\title{
A three generation family with fibrodysplasia ossificans progressiva
}

\author{
J M Connor, H Skirton, P W Lunt
}

\begin{abstract}
A family with five persons affected with fibrodysplasia ossificans progressiva (myositis ossificans progressiva) in three generations is described. This is the first well documented three generation family with this condition and provides further evidence for autosomal dominant inheritance. A wide range of phenotypic severity is apparent, from disabling ectopic bone formation and premature death to an asymptomatic adult with characteristic big toe malformations.

(f Med Genet 1993;30:687-9)
\end{abstract}

Fibrodysplasia ossificans progressiva (FOP, is a rare disorder in which physical handicap owing to progressive soft tissue ossification accompanies characteristic skeletal malformations. ${ }^{1}$ Over 600 patients have been described and attempted total ascertainment in the UK found a minimum point prevalence of one patient per 1.64 million of the population. ${ }^{2}$ The sex ratio is equal and autosomal dominant inheritance is favoured on the basis of concordant monozygotic twins, ${ }^{34}$ a paternal age effect for presumed new mutations, ${ }^{256}$ and several instances of parent to child transmission, including male to male transmission. ${ }^{78}$ Only one three generation family with FOP has been published. Gaster ${ }^{9}$ in 1905 mentioned a family with a grandfather, father, and three sons affected in a discussion at a medical meeting but never published the family in further detail. We thus wish to describe a contemporary three generation family with FOP.

\section{Case report}

Duncan Guthrie Institute of Medical Genetics, Yorkhill Glasgow G3 8SJ, UK. $\mathrm{J} M$ Connor

Clinical Genetics Service, Taunton and Somerset Hospital, Taunton, Somerset, UK.

H Skirton

Clinical Genetics Unit, Institute of Child Health, Bristol Royal Hospital for Sick Children, Bristol Sick P W Lunt

Correspondence to Professor Connor.

Received 16 February 1993 Accepted 26 March 1993.

The family pedigree is shown in fig 1 . The proband (II.2) was asymptomatic until 22 years of age when she developed jaw fixation

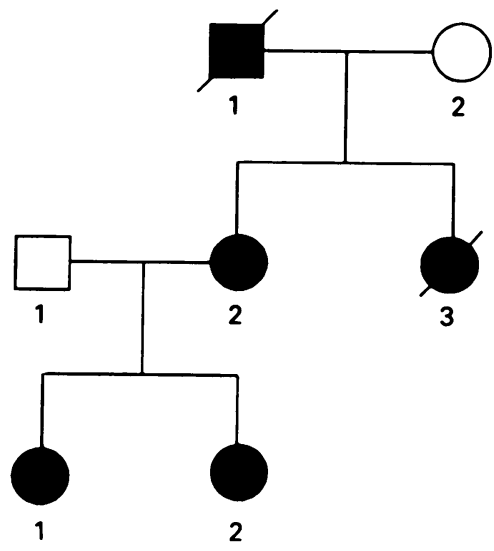
myositis ossificans progressiva, MIM 135100)

after extraction of wisdom teeth. She had a further operation at 23 years of age to replace the right temporomandibular joint with a Bowerman-Conroy titanium prosthesis but jaw fixation recurred one week afterwards. She is now 47 years of age and her only other symptom has been gradually increasing back stiffness in recent years. On examination she has limited neck mobility and jaw fixation but normal mobility at other joints and clinically normal big toes. A radiograph of her cervical spine showed anterior osteophytic bridging of several vertebral bodies (fig 2) and a radiograph of her feet showed symmetrical bony spurs on the medial sides of the heads of the first metatarsals (fig 3).

Her father (I.1, fig 1) had also been asymptomatic until he developed back and neck

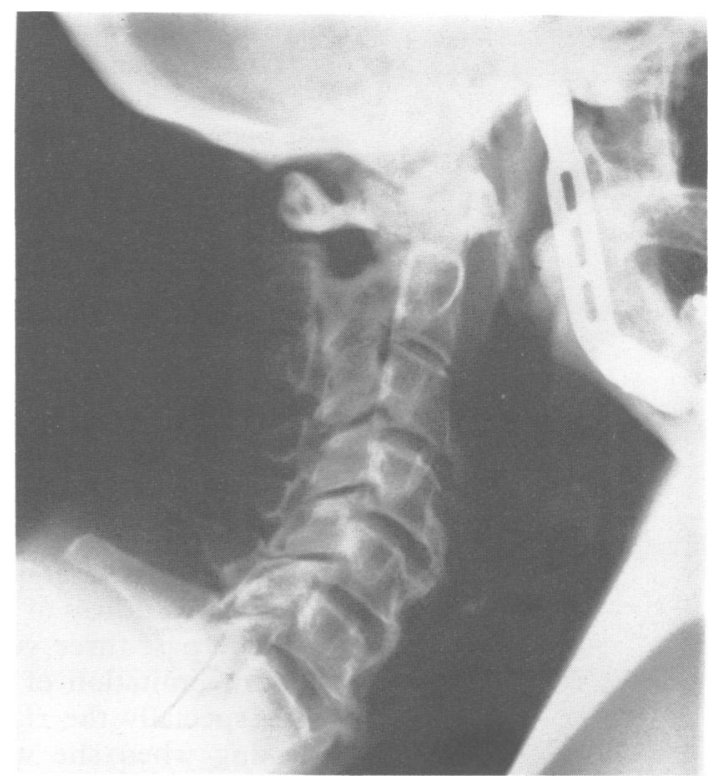

Figure 2 Radiograph of the cervical spine of II.2.

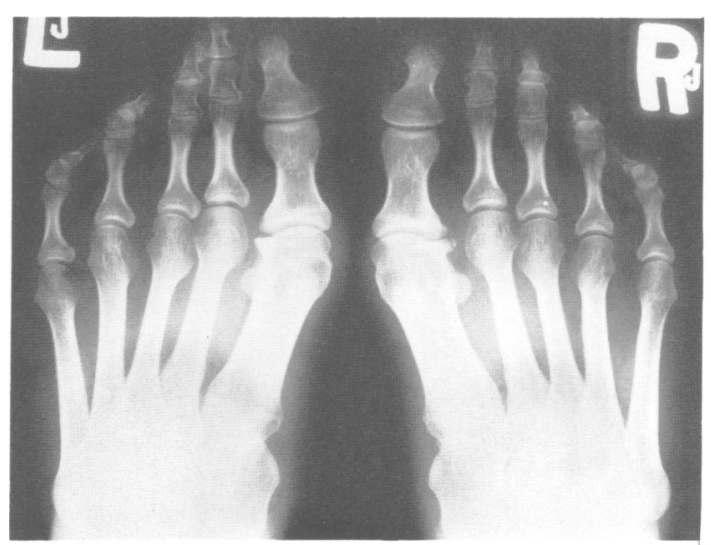

Figure 3 Radiograph of both feet of II.2.

Figure 1 Pedigree of the family. 
stiffness after trauma. His jaw also became fixed after trauma and by his forties he had developed a limp. He remained ambulant (with the use of a stick) until his early seventies and died at 72 years of age from a myocardial infarction. The diagnosis of FOP was confirmed at the Royal National Orthopaedic Hospital, London and some of his $x$ rays are still held in their radiological museum. These were reviewed and showed normal hands, malformed big toes with superimposed ankylosis (similar to his granddaughter III.1), progressive ankylosis of the cervical spine, and multiple areas of soft tissue ossification.

The proband's sister also had FOP. She was well until 15 years of age when spontaneous swelling of the left leg occurred. A biopsy was performed and the family was told she had a fibrosarcoma. The swelling subsequently subsided but she had limited left knee movements thereafter. In her late teens she developed limitation of movement at the right elbow after trauma. In her twenties she developed a succession of painless lumps on her back. After this she started to walk with a frame. She gradually became more disabled and was bed bound for a year before her death from pneumonia at 28 years of age. Her case records and radiographs are no longer available but her hospital diagnosis at the time of death was myositis ossificans.

The proband's two daughters also have FOP. The older (III.1) has had stiff big toes for many years but is otherwise asymptomatic. Her examination at 24 years of age was normal except for no mobility at the interphalangeal joints of both big toes. Her radiographs showed malformed halluces with bony spurs on the medial sides of the heads of the first metatarsals and ankylosis of the hallucal interphalangeal joints (fig 4). Her radiograph of the cervical spine was normal (fig 5 ). The younger daughter was well until 13 years of age when she first noted painful lumps on her back. These appeared over several hours and resolved over several days. Subsequently she has noted stiffness of her neck and lower back. For the past three years she has experienced painful limitation of movement of both hips but especially the right. She noted pain and clicking when she walks and this had been associated with occasional painful locking. On examination at 23 years of age she had palpable

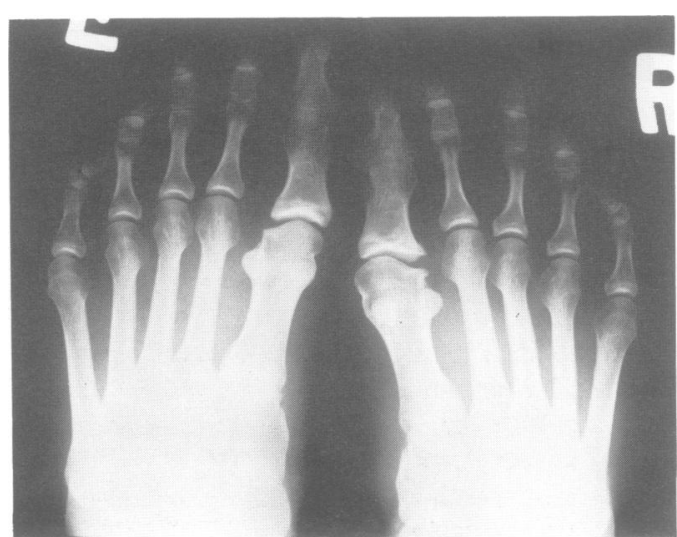

Figure 4 Radiograph of both feet of III.1.

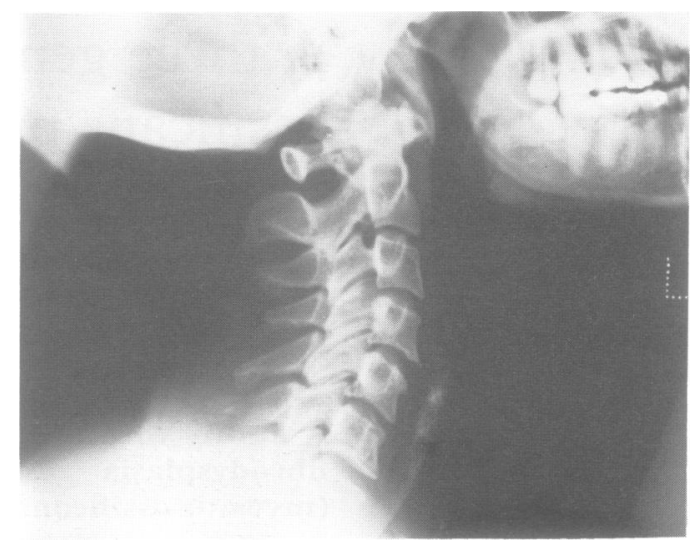

Figure 5 Radiograph of the cervical spine of III.1.

ectopic bone in the left lumbar region, limited mobility of the neck and spine, and painful limitation of all movements at both hips (right more than left), but clinically normal big toes. Her radiographs showed malformed big toes with medial spurs on the heads of the first metatarsals (fig 6), an ectopic bony bar in the left lumbar region (fig 7), a normal cervical spine (fig 8), and abnormalities of both hips with multiple osteochondromata and deformed femoral heads (fig 9).

\section{Discussion}

This family shows many typical features of FOP but has two unusual features and one

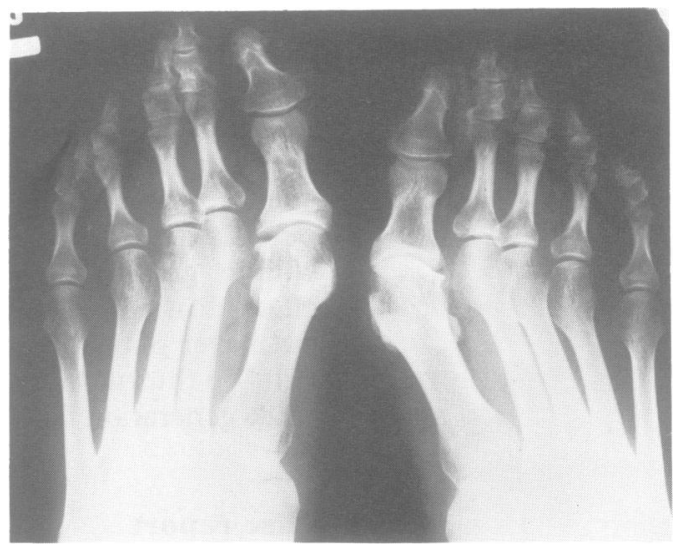

Figure 6 Radiograph of both feet of III.2.

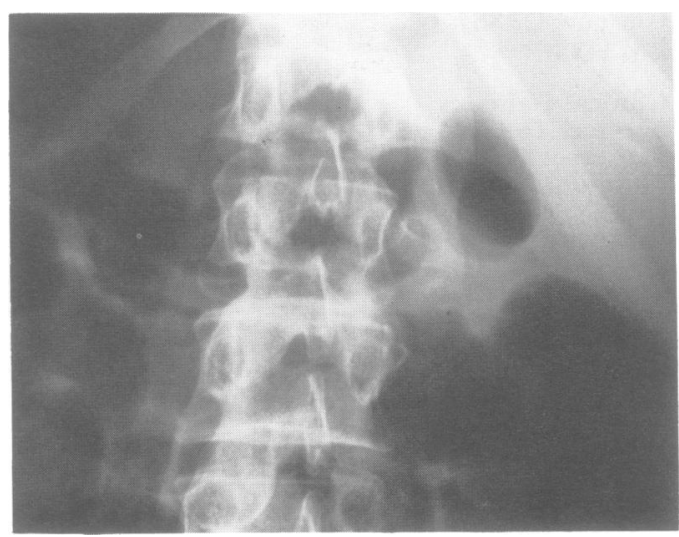

Figure 7 Radiograph of the upper lumbar spine of III.2. 


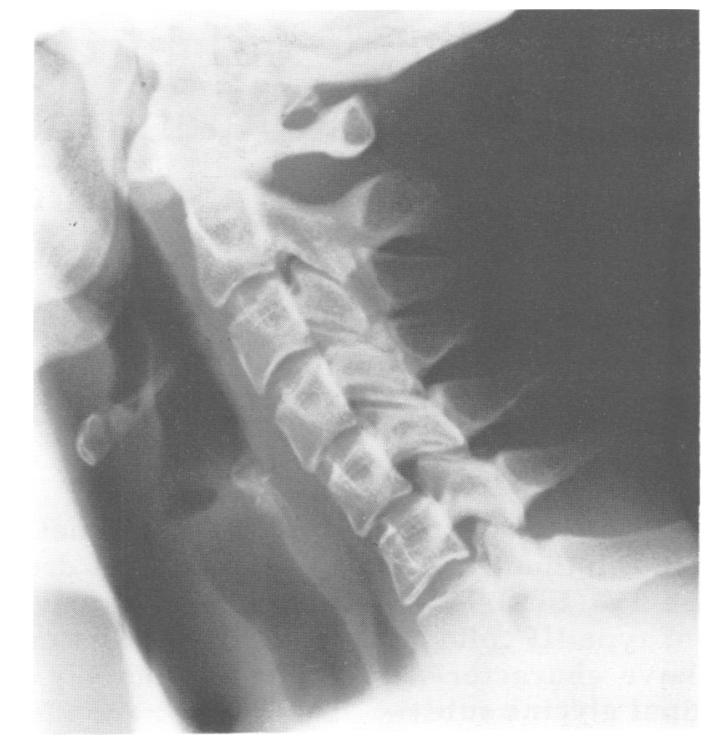

Figure 8 Radiograph of the cervical spine of III.2.

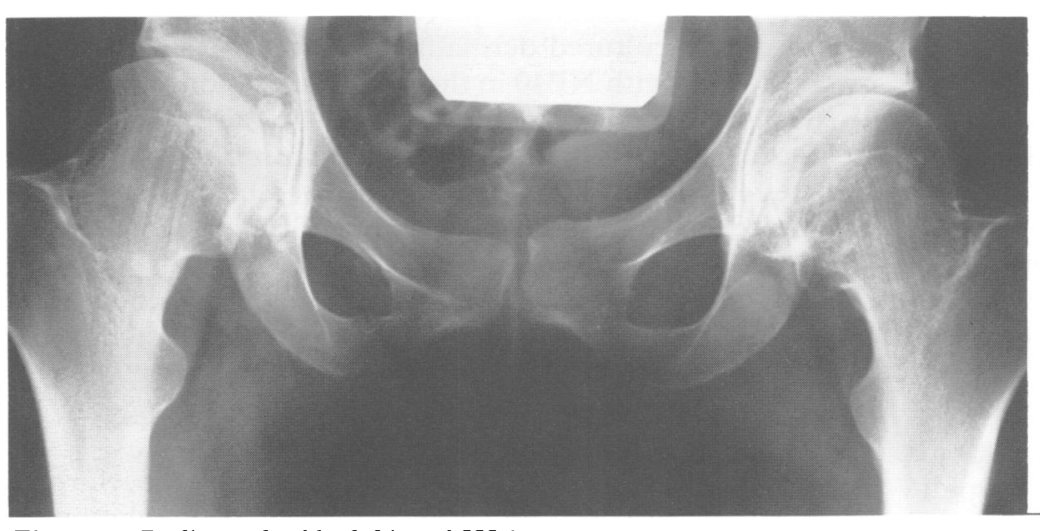

Figure 9 Radiograph of both hips of III.2.

atypical feature. Typical features include the big toe malformations, the pattern of ectopic bone formation, the relationship of ectopic bone to trauma (including surgery), and the misdiagnosis of early ossifying lesions as a fibrosarcoma. The unusual features are the normal cervical spine radiographs in adulthood in III.1 and III. 2 and the mild disability in adulthood in I.1, II.2, III.1, and III.2. The atypical feature is the presence of symptomatic multiple hip osteochondromata in III.2.

In the most common big toe malformation in FOP there is a single phalanx which results in a short big toe which often has valgus deviation. ${ }^{10}$ Less commonly other malformations are found which include normal sized big toes which may later become stiff owing to ankylosis. ${ }^{10}$ Each member of this family (where radiographs were available for study) showed the same malformation of the first metatarsal with superimposed ankylosis in I.1 and III.2. As in these patients, the malformations in FOP are characteristically symmetrical whereas the ectopic bone formation is asymmetrical and unpredictable. Involvement of the paraspinal muscles and extra-articular tissues of the jaw is common with resulting spinal stiffness and jaw fixation. ${ }^{10}$
Hall and Sutcliffe ${ }^{11}$ described abnormal cervical vertebrae with small bodies, enlarged pedicles and short 'massive' spinous processes in eight children with FOP. Similar changes were found in four of eight other children with FOP and in adult life variable fusion of the cervical vertebrae was a consistent feature. ${ }^{12}$ This fusion was first noted between adjacent neural arches in late childhood but also involved the vertebral bodies in some adult patients. The normal cervical spines in adulthood in patients III.1 and III. 2 are thus unusual and probably reflect the generally benign clinical course in these patients.

In general patients are severely handicapped owing to ectopic bone formation. Most patients have severe limitation of the spine and shoulders by 10 years of age, one or both hips are involved by 20 years of age, and most patients are chair or bed bound by 30 years of age. ${ }^{10}$ The natural history in II.3 is thus fairly typical whereas the four other affected subjects in this family have had considerably milder clinical courses. This may reflect the nature of the underlying mutation or factors affecting variable expression of this dominant trait. As yet these factors are unknown and no medical treatment has been shown to influence the long term prognosis. ${ }^{1}$

Multiple osteochondromata have not previously been described in the hip joint in FOP. Usually these represent metaplastic cartilaginous nodules derived from the synovial membrane (synovial osteochondromatosis). ${ }^{13}$ Synovial osteochondromatosis most commonly occurs in young or middle aged adults and the most frequent areas of involvement are the knee joint, the hip, elbow, and shoulder.

The genetic defect causing FOP and its chromosomal regional localisation are currently unknown and linkage studies in families such as this will assist with exclusion mapping of possible candidate genes for this disorder.

We wish to thank Dr D J Stoker of the Royal National Orthopaedic Hospital Trust for kindly providing us with copies of radiographs of I.1.

1 Connor JM. Fibrodysplasia ossificans progressiva. In: Royce PM, Steinmann B. Connective tissue and its heritable disorders. Molecular, genetic and medical aspects. New York: Wiley-Liss, 1992:603-11.

2 Connor JM, Evans DAP. Genetic aspects of fibrodysplasia ossificans progressiva. $\mathcal{f}$ Med Genet 1982;19:35-9.

3 Eaton WL, Conkling WS, Daeschner CW. Early myositis ossificans progressiva occurring in homozygotic twins. A ossificans progressiva occurring in homozygotic twins. A
clinical and pathologic study. $f$ Pediatr 1957;50:591-8.

4 Vastine JH, Vastine MF, Arango O. Myositis ossificans progressiva in homozygotic twins. AfR 1948;59:204-12.

5 Tunte W, Becker PE, von Knorre GV. Zur Genetik der Tunte W, Becker PE, von Knorre GV. Zur Genetik der
Myositis ossificans progressiva. Humangenetik 1967;4:320-51.

6 Rogers JG, Chase GA. Paternal age effect in fibrodysplasia ossificans progressiva. $\mathcal{F}$ Med Genet 1979;16:147-8.

7 Burton-Fanning FW, Vaughan AL. A case of myositis ossificans. Lancet 1901;ii:849-50.

8 Logacev KD. Progressive ossification of muscles. Klin Med (Mosk) 1964;42:106-10.

9 Gaster A. Discussion in meeting in West London MedicoChirurgical Society, 7 October 1904. West London Med $\mathcal{F}$ 1905;10:37.

10 Connor JM, Evans DAP. Fibrodysplasia ossificans progressiva. The clinical features and natural history of 34 patients. $\mathcal{F}$ Bone foint Surg $(B r)$ 1982;64:76-83.

$11 \mathrm{Hall} C \mathrm{CM}$, Sutcliffe J. Fibrodysplasia ossificans progressiva. Ann Radiol (Paris) 1979;22:119-23.

12 Connor JM, Smith R. The cervical spine in fibrodysplasia ossificans progressiva. Br $\mathcal{f}$ Radiol 1982;55:492-6.

13 Milgram JW. Synovial osteochondromatosis: a histopathological study of 30 cases. $f$ Bone foint Surg $(\mathrm{Am})$ logical study of 